\title{
Use of the Acrylonitrile Haemoglobin Adduct N-(2-cyanoethyl)valine as a Biomarker Of Compliance in Smokers Switching to Tobacco Heating Products
}

Oscar M. Camacho, Michael McEwan, Nathan Gale, Nikola Pluym, Max Scherer, George Hardie, and James Murphy

Objective: To investigate the use of blood levels of $\mathrm{N}-(2$ cyanoethyl)valine (CEVal), a haemoglobin adduct of acrylonitrile, to assess lack of compliance with smoking cessation.

Methods: We report CEVal concentrations and percentiles over time for 2 cessation groups, compliance was established using NNAL concentrations. CEVal half-life was estimated. Optimal thresholds were calculated based on receiver operating characteristics analysis.

Results: At 180 days, among 67 participants in the continued smoking group and 159 assigned to smoking cessation or sole THP use. CEVal half-life was estimated to be approximately 30 days and the optimal thresholds were for NNAL at $40 \mathrm{pg} / \mathrm{mL}$ and CEVal at $35 \mathrm{pmol} / \mathrm{g}$ globin (81\% sensitivity and specificity). Conclusions: A new generation of biomarkers of compliance is required, specific to the new generation of nicotine products. Methodological validation and standardisation could allow robust assessment of effects across clinical and observational studies while promoting comparability between studies. CEVal could play an important role as biomarker of compliance for smoking cessation and switching studies.

Key words: tobacco heating product; CEVal; protocol compliance; smoking cessation, biomarker

Oscar M Camacho, Statistics and Modeling Manager, Michael McEwan, Senior Scientist (Clinical Research), Nathan Gale, Senior Scientist (Clinical Research), George Hardie, Clinical Research Manager, Research and Development, British American Tobacco (Investments) Limited, Southampton, UK.

James Murphy, Executive Vice-President of Scientific and Regulatory Affairs, Reynolds American Inc, $401 \mathrm{~N}$ Main Street, Winston-Salem, NC, USA

Nikola Pluym, Technical Director, Max Scherer, General Manager, ABF Analytisch-

Biologisches Forschungslabor GmbH, Planegg, Germany

Correspondence Dr Michael McEwan; Mike_McEwan@bat.com 
Cigarette smoking causes numerous human diseases including lung cancer, chronic obstructive pulmonary disease, and cardiovascular disease. ${ }^{1}$ The principal cause is inhalation exposure to a high number of smoke toxicants emitted when tobacco is combusted. ${ }^{1-3}$ In a review of tobacco harm reduction data, the Royal College of Physicians concluded that use of nicotine and tobacco products that support sustained displacement of combustible cigarette smoking can reduce smoking related harm by reducing and/or eliminating toxicant exposure. ${ }^{4}$

Tobacco heating products (THPs) electronically warm tobacco to temperatures far below cigarettes $\left(\mathrm{eg}, 240^{\circ} \mathrm{C}\right.$ instead of $\left.900^{\circ} \mathrm{C}\right),{ }^{5}$ releasing an aerosol which, for some products, has been shown to have substantially lower numbers and yields of toxicants than cigarette smoke. ${ }^{6-8}$ In a short-term switching study, among smokers who switched to a THP for 5 days in a confined setting, most of the biomarkers of exposure to tobacco smoke were reduced to levels similar to those seen in participants who refrained completely from using any tobacco products. ${ }^{9}$

To assess long-term changes in exposure and risk, we have embarked on a 1-year THP switching study to assess biomarkers of exposure and biomarkers of potential harm in volunteers switching from combustible cigarettes to a THP branded as glo or smoking cessation. ${ }^{10}$ Although participants are encouraged to use glo exclusively, with most consumption taking place at home there is a risk of some degree of dual use or relapse to smoking, particularly given the study duration. In a 90-day THP switching study, selfreporting and carbon monoxide breath tests indicated that $39(49 \%)$ of 80 participants were not fully compliant with smoking abstinence. ${ }^{11}$ If undetected, non-compliance could underestimate changes in biomarker levels.

Biochemical verification of tobacco use and abstinence is recommended where possible in clinical studies, but assessments are hindered by lack of specific, validated biomarkers for this purpose that can be detected long enough in humans to evaluate sustained tobacco use abstinence. ${ }^{12}$ The most obvious candidate biomarkers to assess compliance would be biomarkers of exposure to tobacco specific nitrosamines (TSNAs), due to their specificity to tobacco smoke exposure. For smoking cessation studies 4-(methylnitrosamino)-1-(3 pyridyl)1-butanol (NNAL) has been used to verify smoking abstinence. ${ }^{13}$ However, as THPs contain tobacco, their emissions contain TSNAs, ${ }^{6,14}$ albeit at much lower levels than in cigarette smoke. ${ }^{6}$ This makes biomarkers such as NNAL unsuitable for monitoring compliance. Halflife of NNAL in urine is relatively short - around 10 days - and concentrations can be detectable for up to roughly 8 - 12 weeks after smoking cessation, ${ }^{15}$ which could limit its usability for longer term studies. Additionally, NNAL is often used as a primary endpoint in smoking related studies and this therefore prevents its use to assess compliance within those studies.

We would like to suggest N-(2 cyanoethyl)valine (CEVal) in blood as a biomarker of long-term smoking-cessation compliance in participants using THPs. It is a haemoglobin $\mathrm{Hb})$ adduct of acrylonitrile, which is a major tobacco smoke constituent (mean estimated level $34 \mu \mathrm{g}$ [range 19-50 $\mu \mathrm{g}$ ] per cigarette) ${ }^{16}$ and secondhand smoke, ${ }^{17}$ but levels are negligible in THP vapour. ${ }^{6,14}$ CEVal can be detected for up to 120 days ${ }^{18}$ and has good specificity to smoking, with background concentrations of acrylonitrile associated with environmental exposure, including via occupational exposure, generally very low. ${ }^{19-23}$ Therefore, CEVal should be an appropriate biomarker for differentiating smokers and dual users from exclusive users of THPs.

In this manuscript we present our efforts to evaluate CEVal as biomarker of compliance to smoking cessation based on smokers who have quitted all tobacco products. ${ }^{9,10}$ The specificity and sensitivity of CEVal as biomarker of compliance is benchmarked against NNAL biomarker levels in the cessation group. 


\section{METHODS}

\section{Study Design}

This analysis was performed in the participants of a randomised, multicentre, controlled clinical THP switching study. ${ }^{10}$ Eligible participants are healthy male and female current smokers or non-smokers aged 23-55 years, with a history of good general health and no clinically relevant abnormal findings on physical examination, vital signs assessment, electrocardiography, clinical laboratory evaluations or lung function tests. Participants who do not intend to quit smoking were randomized to continued combustible smoking or a commercially available glo THP. Participants with a high intent to quit smoking were assigned to the smoking cessation arm. The study also recruited never smokers as a control group. Full inclusion and exclusion criteria have been published. ${ }^{9,10}$

Smokers were randomly assigned to continue smoking their usual brand of factory manufactured or roll-your-own cigarettes or switch completely to using the glo THP with non-menthol tobacco consumables or to tobacco abstinence. ${ }^{9,10}$ In this ancillary analysis we evaluate those participants in the cessation arm.

All participants are informed that they are free to quit smoking and withdraw from the study at any time. Anyone who decides to quit smoking is directed to appropriate stop smoking services. Complete information about the design of this study has been published previously. 9,10

\section{CEVal Analytical Method}

In the THP switching study, CEVal blood samples were collected from participants in ethylenediaminetetraacetic acid (EDTA) blood collection tubes at days 0, 30, 60, 90, and 180 . Samples were analysed according to a previously described method with minor modifications. ${ }^{24,25}$ Briefly, globin was isolated from washed erythrocytes according to the protocol described by Schettgen et al. ${ }^{24}$ using $5 \mathrm{~mL}$ of the hemolysate. $100 \mathrm{mg}$ of the isolated globin, accurately weighed, were suspended with $1.5 \mathrm{~mL}$ of formamide and vortexed until complete dissolution. After addition of $20 \mu \mathrm{L}$ of the internal standard $\left(\mathrm{d}_{3}-\mathrm{CEV}\right.$ al approx. 130 $\mathrm{pmol} / \mathrm{g}$ globin in formamide), $20 \mu \mathrm{L}$ of $4 \mathrm{M}$ sodium hydroxide, the solution was mixed with $25 \mu \mathrm{L}$ of pentafluorophenylisothiocyanate and derivatized for 3 hours at $80^{\circ} \mathrm{C}$. After liquidliquid extraction with $8 \mathrm{~mL}$ of methyl-tert-butylether the organic phase was evaporated to dryness and reconstituted in $2 \mathrm{~mL}$ toluene and $2 \mathrm{~mL}$ of water. The organic phase was subsequently washed with $2 \mathrm{~mL}$ of $0.1 \mathrm{M}$ sodium carbonate and evaporated to dryness. The residue was dissolved in $100 \mu \mathrm{L}$ toluene and $1 \mu \mathrm{L}$ were injected (splitless mode) into the GCMS/MS system (TQ8050, Shimadzu, Neufahrn, Germany). Chromatographic separation was achieved on an Rxi 5-ms column (30 m x 0.25 mm, $0.5 \mu \mathrm{m})$ (Restek, Bad Homburg, Germany) with helium as carrier gas $(1.45 \mathrm{~mL} / \mathrm{min})$ and a temperature gradient starting at $110{ }^{\circ} \mathrm{C}$ for $1 \mathrm{~min}, 25^{\circ} \mathrm{C} / \mathrm{min}$ to $205^{\circ} \mathrm{C}, 5^{\circ} \mathrm{C} / \mathrm{min}$ to $240{ }^{\circ} \mathrm{C}$ and $30^{\circ} \mathrm{C} / \mathrm{min}$ to $300{ }^{\circ} \mathrm{C}$. Mass spectrometric detection was performed after electron ionization in multiple-reaction monitoring mode (MRM) $(335 \mathrm{~m} / \mathrm{z}$ to $282 \mathrm{~m} / \mathrm{z})$.

Method precision for both intra day and inter day was acceptable, with CV less than $15 \%$. Accuracy for all levels were within the acceptance ranges of 85 to $115 \%$. The lower limit of quantification was estimated to be at $2.0 \mathrm{pmol} / \mathrm{g}$ globin.

\section{NNAL Analytical Method}

Measurements of total NNAL were taken at days $0,30,60,90$, and 180 by LC-MS/MS analysis after solid-phase extraction (SPE) according to Kavvadias et al. ${ }^{26}$ with minor modifications. Briefly, $8 \mathrm{~mL}$ of urine were incubated overnight with $40 \mu \mathrm{L} \beta$-glucuronidase (E.coli $155 \mathrm{U} / \mu \mathrm{L})$ at $\mathrm{pH} 7.2$ after addition of the internal standard $\left(20 \mu \mathrm{L} \mathrm{d}_{3}\right.$-NNAL; 25 $\mathrm{ng} / \mathrm{mL}$ in water) and subsequently purified by SPE according to Kavvadias et al. ${ }^{26} 10 \mu \mathrm{L}$ of 
the final extract were injected into the LC-MS/MS. Chromatography was performed on an Acquity UPLC HSS T3 column $(100 \times 2.1 \mathrm{~mm} ; 1.8 \mu \mathrm{m})$ (Waters, Eschborn, Germany) at 45 ${ }^{\circ} \mathrm{C}$ with a flow rate of $0.6 \mathrm{~mL} / \mathrm{min}$. Gradient elution was accomplished using $0.1 \%$ ammonium acetate in water (A) and $0.1 \%$ ammonium acetate in acetonitrile (B) as follows: 0-1 min: $90 \%$ A, $1-2$ min: $90-50 \%$ A, 2-4 min: $50 \%$ A, $4.1 \min : 90 \%$ A, 7 min: $90 \%$ A. Positive electrospray ionization was applied and the MS/MS system was run in MRM using the mass transitions as published. ${ }^{26}$

\section{Statistical Analysis}

This analysis included participants in the THP switching study who had data available to 180 days. ${ }^{27}$ Demographics data reported are: age; sex; and race. Summary statistics are presented for blood CEVal concentrations at baseline, including number of observations, mean, standard deviation, range, and percentiles.

Change in CEVal over time was summarised for each time-point as mean, standard deviation, range, and number of observations.

CEVal percentiles over time were estimated using robust quantile regression with logtransformed CEVal as the dependent variable and time in days since study onset as an explanatory variable. Quantile confidence intervals were calculated using the rank method ${ }^{28}$ and the upper $95 \%$ confidence limits are presented.

The 5th, 10th, 25th, 50th, 75th, 90th and 95th percentiles of the CEVal concentrations at days $0,30,60,90$ and 180 were estimated for participants with confirmed smoking cessation using NNAL biomarker levels. We used 2 thresholds as criteria for smoking cessation compliance. First, we used concentrations below the limit of quantification $(<$ LoQ). As concentrations $<10 \mathrm{pg} / \mathrm{mL}$ of NNAL have also been shown to discriminate between smokers and non-smokers exposed to background levels of NNAL (ie, secondhand smoke), ${ }^{29-}$ ${ }^{31}$ we also set the criterion of NNAL concentrations $<$ LoQ or $<10 \mathrm{pg} / \mathrm{mL}$ NNAL, whichever was highest. CEVal estimates are calculated based on concentration values up to the last day of recorded compliance according to NNAL thresholds.

CEVal half-life was calculated using a repeated measures model in the log-transformed CEVal data complying with NNAL $<$ LoQ or $<10 \mathrm{pg} / \mathrm{mL}$ (whichever highest) with time as independent variable.

A confusion matrix was created to assess classifications based on the 2 NNAL criteria. The CEVal threshold to discriminate between smokers and non-smokers was set at $35 \mathrm{pmol} / \mathrm{g}$ globin which is a conservative threshold to account for potential occupational or environmental exposure. ${ }^{32,33}$ To create the confusion matrix, the last observation for each participant at either day 60,90 or 180 was used, adding to a total of 135 observations.

Based on a receiver operation characteristics (ROC) analysis, optimal thresholds for

NNAL were calculated to minimize the difference between sensitivity and specificity considering NNAL compliance boundaries at 10,25 and $40 \mathrm{pg} / \mathrm{mL} .^{12}$

All analyses were performed with SAS version 9.4, SAS Institute, Cary, US.

\section{RESULTS}

At the time of this analysis, 226 participants were enrolled in the THP switching study and had data reported up to day 180 . Of these, 67 were in the continued smoking group and 159 were in the cessation groups. Characteristics of participants in this analysis were well matched across study groups (Table 1). At baseline, overall CEVal concentrations in the continued smoking and smoking cessation groups were similar although, by percentile, values were slightly lower in the cessation group up to the 90th percentile (Table 2). 
Table 1: Baseline Characteristics of Participants in the Tobacco Heating Product 1-Year Switching Study Who Continued Smoking or With Confirmed Cessation

\begin{tabular}{cccccccccccc}
\hline \multirow{2}{*}{ Group } & $\begin{array}{c}\text { No. } \\
\text { partici- } \\
\text { pants }\end{array}$ & Mean & SD & Min & Max & Male & Female & White & Black & Asian & Other \\
\hline $\begin{array}{c}\text { Continued } \\
\text { smoking }\end{array}$ & 67 & 36.9 & 9.87 & 23 & 55 & $59.7 \%$ & $40.3 \%$ & $88.1 \%$ & $4.5 \%$ & $6.0 \%$ & $1.5 \%$ \\
\hline Cessation & 159 & 38 & 8.99 & 23 & 55 & $59.1 \%$ & $40.9 \%$ & $90.6 \%$ & $2.5 \%$ & $2.5 \%$ & $4.4 \%$ \\
\hline $\begin{array}{c}\text { Cessation } \\
\text { compliant (NNAL } \\
<\text { LoD) }\end{array}$ & 54 & 38 & 8.99 & 24 & 55 & $63.0 \%$ & $37.0 \%$ & $85.2 \%$ & $3.7 \%$ & $3.7 \%$ & $7.4 \%$ \\
\hline $\begin{array}{c}\text { Cessation } \\
\text { compliant (NNAL } \\
<\text { LoD or }<10 \\
\text { pg/mL) }\end{array}$ & 83 & 37 & 8.84 & 23 & 55 & $58.0 \%$ & $42.0 \%$ & $89.2 \%$ & $2.4 \%$ & $2.4 \%$ & $6.0 \%$ \\
\hline
\end{tabular}

Abbreviations: LoD, limit of detection; max, maximum; min, minimum; NNAL, 4-(methylnitrosamino)-1-(3pyridyl)-1-butanol; SD, standard deviation.

Table 2: CEVal Percentiles at Baseline by Study Group and NNAL Criterion for Cessation

\begin{tabular}{|c|c|c|c|c|c|c|c|c|c|c|c|}
\hline \multirow[t]{2}{*}{ Group } & \multicolumn{4}{|c|}{$\begin{array}{l}\text { CEVal concentration (pmol/g } \\
\text { globin) }\end{array}$} & \multicolumn{7}{|c|}{ Percentile (pmol/g globin) } \\
\hline & Mean & SD & Min & Max & $5^{\text {th }}$ & $10^{\text {th }}$ & $25^{\text {th }}$ & $50^{\text {th }}$ & $75^{\text {th }}$ & $90^{\text {th }}$ & $95^{\text {th }}$ \\
\hline Continued smoking $(n=67)$ & 128 & 57.6 & 35 & 279 & 41 & 63 & 85 & 125 & 169 & 214 & 245 \\
\hline Cessation group $(n=159)$ & 127 & 67.0 & 4 & 384 & 31 & 48 & 78 & 122 & 162 & 213 & 253 \\
\hline $\begin{array}{c}\text { Cessation compliant } \\
(\mathrm{NNAL}<\mathrm{LoD} ; \mathrm{n}=54)\end{array}$ & 101 & 67.5 & 13 & 306 & 14 & 32 & 57 & 89 & 124 & 177 & 253 \\
\hline $\begin{array}{l}\text { Cessation compliant } \\
(\mathrm{NNAL}<\mathrm{LoD} \text { or }<10 \\
\text { pg } / \mathrm{mL} ; \mathrm{n}=83 \text { ) }\end{array}$ & 108 & 63.4 & 4 & 306 & 24 & 38 & 58 & 99 & 140 & 180 & 245 \\
\hline
\end{tabular}

Abbreviations: CEVal, N-(2 cyanoethyl)valine; Hb, haemoglobin; LoD, limit of detection; NNAL, 4(methylnitrosamino)-1-(3-pyridyl)-1-butanol. According to the NNAL<LoD criterion: 4 participants were compliant at Day 30, 8 at Day 60,17 at Day 90 and 25 at Day 180. Using the whichever is higher (NNAL $<$ LoD or $<10 \mathrm{pg} / \mathrm{mL}$ ) criterion, 5 participants were compliant at Day 30, 8 at Day 60, 30 at Day 90 and 40 at Day 180.

Of 159 participants in the smoking cessation group, NNAL $<$ LoQ data were available from 223 observations across all 5 timepoints and 54 (34\%) participants were deemed to be compliant for 2 or more study timepoints. Based on NNAL $<$ LoQ criterion, 4 participants were compliant at Day 30, 8 at Day 60,17 at Day 90 and 25 at Day 180. For the NNAL $<$ LoQ or $<10 \mathrm{pg} / \mathrm{mL}$ criterion, 351 observations were available and $83(52 \%)$ participants were categorised as being complaint. Based on this last criterion, 5 participants were compliant at Day 30, 8 at Day 60, 30 at Day 90 and 40 at Day 180. Descriptive statistics and percentiles for CEVal concentrations at baseline for the continued smoking arm and for the cessation group verified according to each NNAL criterion are displayed in Table 2, showing comparable values at baseline across groups.

The average half-life for CEVal was estimated at approximately 30 days from cessation (30.37). Our results suggest similar level of CEVal concentrations across time independently of the NNAL based classification approach. Both quantile regressions point to $95 \%$ of participants reaching day 180 with CEVal concentrations below $25 \mathrm{pmol} / \mathrm{g}$ globin and with point estimates for the $90^{\text {th }}$ percentile 3-7 pmol/g globin. However, it is worth noting that the confidence limits of the CEVal estimates widen towards the data boundaries, indicating increased uncertainty and emphasising already wide confidence intervals. The upper bounds 
in the 95 th percentile could be as high as $179 \mathrm{pmol} / \mathrm{g}$ globin or $77 \mathrm{pmol} / \mathrm{g}$ globin dependent on the NNAL criterion used (Table 3 ).

Table 3: Estimated Blood CEVal Concentration Percentiles by Smoking Cessation Compliance Criterion and Timepoint

\begin{tabular}{|c|c|c|c|c|c|c|c|c|}
\hline \multirow{2}{*}{$\begin{array}{c}\text { CEVal } \\
\text { (pmol/g globin) } \\
\text { Time-point }\end{array}$} & \multicolumn{4}{|c|}{$\begin{array}{c}\text { Days since smoking cessation NNAL }<\text { LoQ } \\
\text { criterion }(\mathrm{N}=54)\end{array}$} & \multicolumn{4}{|c|}{$\begin{array}{c}\text { Days since smoking cessation NNAL }<\text { LoQ or } \\
<10 \mathrm{pg} / \mathrm{mL} \text { criterion }(\mathrm{N}=83)\end{array}$} \\
\hline & 30 & 60 & 90 & 180 & 30 & 60 & 90 & 180 \\
\hline $\mathrm{n}$ & 52 & 50 & 42 & 25 & 80 & 78 & 70 & 40 \\
\hline mean & 70.6 & 38.4 & 14.8 & $<$ LoQ & 74.0 & 38.5 & 15.5 & $<$ LoQ \\
\hline SD & 48.7 & 27.9 & 14.5 & 24.5 & 44.9 & 25.4 & 14.7 & 23.3 \\
\hline $\max$ & 217.6 & 129.6 & 88.3 & 121.4 & 217.6 & 121.6 & 88.3 & 121.4 \\
\hline $\min$ & $<$ LoQ & $<$ LoQ & $<$ LoQ & $<$ LoQ & $<$ LoQ & $<$ LoQ & $<\mathrm{LoQ}$ & $<\mathrm{LoQ}$ \\
\hline 95th Percentile & $169(355)$ & $116(309)$ & $79(270)$ & 25 (179) & $161(214)$ & $110(174)$ & $76(142)$ & $24(77)$ \\
\hline 90 $0^{\text {th }}$ Percentile & $117(236)$ & $55(174)$ & $26(129)$ & $3(52)$ & $117(183)$ & 67 (133) & $38(96)$ & $7(36)$ \\
\hline $75^{\text {th }}$ Percentile & 80 (107) & $38(55)$ & $18(28)$ & $2(4)$ & $86(107)$ & $43(57)$ & $22(31)$ & $3(5)$ \\
\hline $50^{\text {th }}$ Percentile & $53(67)$ & $25(34)$ & $12(17)$ & $<$ LoQ & $59(71)$ & $27(35)$ & $12(18)$ & $<$ LoQ \\
\hline $25^{\text {th }}$ Percentile & $33(44)$ & $16(21)$ & $7(11)$ & $<$ LoQ & $35(44)$ & $16(21)$ & $7(10)$ & $<\mathrm{LoQ}$ \\
\hline $10^{\text {th }}$ Percentile & $20(28)$ & $10(14)$ & $5(7)$ & $<$ LoQ & $24(28)$ & $11(14)$ & $5(7)$ & $<$ LoQ \\
\hline $5^{\text {th }}$ Percentile & $11(18)$ & $6(11)$ & $3(6)$ & $<$ LoQ & $16(21)$ & $8(11)$ & $4(6)$ & $<$ LoQ \\
\hline
\end{tabular}

Percentiles values presented are estimates of blood CEVal from quantile regressionand upper 95\% confidence limits in parenthesis. NNAL, 4-(methylnitrosamino)-1-(3-pyridyl)-1-butanol. LoQ, limit of quantification. Ceval $\mathrm{LoQ}=2 \mathrm{pmol} / \mathrm{g}$ globin. According to the NNAL<LoD criterion: 4 participants were compliant at Day 30, 8 at Day 60, 17 at Day 90 and 25 at Day 180. Using the whichever is higher (NNAL $<$ LoD or $<10 \mathrm{pg} / \mathrm{mL}$ ) criterion, 5 participants were compliant at Day 30, 8 at Day 60, 30 at Day 90 and 40 at Day 180.

Abbreviations: CEVal, N-(2 cyanoethyl)valine; LoD, limit of detection; LoQ limit of quantification; SD, Standard deviation.

Classification matrix based on CEVal and NNAL suggest good sensitivity for noncompliant volunteers with more than $95 \%$ of observations classified as non-compliant by CEVal also being classified as non-compliant using NNAL (Table 4). However, specificity was significantly lower, with only $32.6 \%$ of volunteers classified as compliant using the CEVal method also classified as complaint using NNAL $<$ LoQ criterion. Specificity marginally improves to $51.7 \%$ when the $10 \mathrm{pg} / \mathrm{mL}$ threshold is applied (Table 4). In fact, optimal cutpoint from ROC analyses suggest that, for a threshold of $10 \mathrm{pg} / \mathrm{mL}$ for NNAL, according to a difference optimisation criterion, a limit at $5.2 \mathrm{pmol} / \mathrm{g}$ globin for CEVal offers optimal threshold at approximately $71 \%$ sensitivity and specificity. Increasing the thresholds for NNAL provide higher agreement. With NNAL at $25 \mathrm{pg} / \mathrm{mL}$ the ROC analysis suggest $14.3 \mathrm{pmol} / \mathrm{g}$ globin as optimal threshold for CEVal with $74 \%$ sensitivity and specificity. With NNAL at $40 \mathrm{pg} / \mathrm{mL}$ the optimal threshold for CEVal would be $35.2 \mathrm{pmol} / \mathrm{g}$ globin and $81 \%$ sensitivity and specificity (Table 5). 
Table 4: Confusion Matrix

\begin{tabular}{|c|c|c|c|c|c|c|c|c|c|c|}
\hline \multirow{3}{*}{ Criterion } & \multicolumn{10}{|c|}{ CEVal<35 pmol/g globin } \\
\hline & \multicolumn{5}{|c|}{ Non-Compliant } & \multicolumn{5}{|c|}{ Compliant } \\
\hline & $\begin{array}{c}\text { Number (Col\% / } \\
\text { Row\%) }\end{array}$ & Mean & SD & Min & $\operatorname{Max}$ & $\begin{array}{c}\text { Number (Col \% / } \\
\text { Row \%) }\end{array}$ & Mean & SD & Min & Max \\
\hline \multicolumn{11}{|c|}{ NNAL $<$ LoQ } \\
\hline $\begin{array}{c}\text { Non-compliant } \\
\text { CEVal (pmol/g globin) } \\
\text { NNAL }(\mathrm{pg} / \mathrm{mL}) \\
\text { Compliant }\end{array}$ & $45(97.8 \%$ / 42.9\%) & $\begin{array}{l}115 \\
101\end{array}$ & $\begin{array}{l}59.3 \\
125\end{array}$ & $\begin{array}{l}35 \\
7.5\end{array}$ & $\begin{array}{l}261 \\
669\end{array}$ & $60(67.4 \% / 57.1 \%)$ & $\begin{array}{c}7.6 \\
20.9\end{array}$ & $\begin{array}{c}9.2 \\
13.3\end{array}$ & $\begin{array}{c}<\mathrm{LoQ} \\
2.8\end{array}$ & $\begin{array}{l}33.7 \\
53.8\end{array}$ \\
\hline $\begin{array}{l}\text { CEVal (pmol/g globin }) \\
\text { NNAL }(\mathrm{pg} / \mathrm{mL})\end{array}$ & $1(2.2 \% / 3.3 \%)$ & - & $\begin{array}{l}- \\
-\end{array}$ & $\begin{array}{c}121 \\
<\mathrm{LoQ}\end{array}$ & $\begin{array}{c}121 \\
<\mathrm{LoQ}\end{array}$ & $29(32.6 \% / 96.7 \%)$ & $\begin{aligned} & 3.8 \\
< & \mathrm{LoQ}\end{aligned}$ & $\begin{array}{l}6.2 \\
6.3\end{array}$ & $\begin{array}{l}<\mathrm{LoQ} \\
<\mathrm{LoQ}\end{array}$ & $\begin{array}{c}25.1 \\
16\end{array}$ \\
\hline \multicolumn{11}{|c|}{ NNAL $<$ LoQ or $<10 \mathrm{pg} / \mathrm{mL}$} \\
\hline $\begin{array}{c}\text { Non-compliant } \\
\text { CEVal (pmol/g globin) } \\
\text { NNAL }(\mathrm{pg} / \mathrm{mL}) \\
\text { Compliant }\end{array}$ & $44(95.7 \% / 50.6 \%)$ & $\begin{array}{l}116 \\
103\end{array}$ & $\begin{array}{c}59.9 \\
125.9\end{array}$ & $\begin{array}{c}35 \\
10.3\end{array}$ & $\begin{array}{l}261 \\
669\end{array}$ & $43(43.3 \% / 49.4 \%)$ & $\begin{array}{l}8.2 \\
27\end{array}$ & $\begin{array}{c}9.7 \\
10.6\end{array}$ & $\begin{array}{c}<\mathrm{LoQ} \\
11.7\end{array}$ & $\begin{array}{l}33.7 \\
53.8\end{array}$ \\
\hline $\begin{array}{c}\text { CEVal (pmol/g globin) } \\
\text { NNAL }(\mathrm{pg} / \mathrm{mL})\end{array}$ & $2(4.3 \% / 4.2 \%)$ & $\begin{array}{c}104 \\
<\text { LoQ }\end{array}$ & $\begin{array}{c}25.0 \\
5.3\end{array}$ & $\begin{aligned} & 86 \\
< & \mathrm{LoQ}\end{aligned}$ & $\begin{array}{l}121 \\
7.5\end{array}$ & $46(51.7 \% / 95.8 \%)$ & $\begin{aligned} & 4.6 \\
< & \mathrm{LoQ}\end{aligned}$ & $\begin{array}{l}6.8 \\
5.2\end{array}$ & $\begin{array}{l}<\mathrm{LoQ} \\
<\mathrm{LoQ}\end{array}$ & $\begin{array}{c}25.1 \\
16\end{array}$ \\
\hline
\end{tabular}

Classifications of based on CEVal $35 \mathrm{pmol} / \mathrm{g}$ globin. Threshold and the 2 NNAL criteria for the last observation of each volunteer reaching at least day 60. Mean, SD, min and max are descriptive of CEVal in pmol/g globin. $\mathrm{CEVal}$ LoQ $=2 \mathrm{pmol} / \mathrm{g}$ globin. According to the NNAL $<$ LoD criterion: 4 participants were compliant at Day 30, 8 at Day 60, 17 at Day 90 and 25 at Day 180. Using the whichever is higher (NNAL $<$ LoD or $<10$ pg/mL) criterion, 5 participants were compliant at Day 30, 8 at Day 60, 30 at Day 90 and 40 at Day 180. Abbreviations: CEVal, N-(2 cyanoethyl)valine; Col, column; LoD, limit of detection; LoQ, limit of quantification; Max, maximum; Min, minimum; NNAL, 4-(methylnitrosamino)-1-(3-pyridyl)-1-butanol; SD, standard deviation.

Table 5: Optimal Cut-Offs Based on ROC Analysis

\begin{tabular}{cccc}
\hline $\begin{array}{c}\text { NNAL Compliance } \\
\text { thresholds }\end{array}$ & Sensitivity (\%) & Specificity (\%) & Optimal CEVal cut-off (pmol/g globin) \\
\hline $10 \mathrm{pg} / \mathrm{mL}$ & 70.8 & 71.2 & 5.2 \\
\hline $25 \mathrm{pg} / \mathrm{mL}$ & 74.1 & 74.1 & 14.3 \\
\hline $40 \mathrm{pg} / \mathrm{mL}$ & 80.8 & 80.6 & 35.2 \\
\hline
\end{tabular}

Optimal cut-off based on NNAL thresholds extracted from literature ${ }^{12}$ and according to minimal difference criteria. Abbreviations: CEVal, N-(2 cyanoethyl)valine; NNAL, 4-(methylnitrosamino)-1-(3-pyridyl)-1-butanol; $\mathrm{ROC}$, receiver operation characteristics.

\section{Discussion}

Studies in which participants switch from smoking combustible cigarettes to using novel tobacco and/or nicotine products are important for determining potential changes in risks arising from reduced exposure associated with switching. These types of assessments have become common for e-cigarettes and THPs, indicating reductions in biomarkers of exposure and of biological effect following product switching. ${ }^{9,11,34}$ However, compared with confinement studies where adherence to the study protocol is strictly enforced, compliance in even short ambulatory studies is not typical. ${ }^{11}$ To gain robust information on the effects of switching in real-world settings, long-term ambulatory studies are necessary. Non-compliance, therefore, needs to be accounted for in data analyses to avoid underestimating any effects of switching or smoking cessation.

Participants in switching studies might be wary about reporting non-compliance due to the risk of being removed from the study or simply because of feeling embarrassed that they have not succeeded in completely quitting smoking. Therefore, biochemical verification of 
compliance is needed to assess adherence to the study protocol. ${ }^{12}$ Cotinine in urine or saliva is often used to assess lack of compliance in smoking cessation studies, however, they can only be detected for few hours after nicotine exposure ${ }^{12}$ and clearly they are not useful in the context of nicotine products switching studies. Exhaled CO is another marker used in this context, however, like cotinine, its effectiveness for detecting lack of compliance is limited due to its short lifetime. ${ }^{12}$ NNAL in urine has been suggested as a strong candidate to assess sustained cessation and product switching to those nicotine products which don't contain cured tobacco, more specifically TSNAs, products such NRTs, or e-cigarettes. However, many of these studies use NNAL as an efficacy endpoint for its specificity as biomarker of exposure and its association to lung cancer which prevents its use as compliance marker. ${ }^{35}$ Neither is useful to assess switching to THPs as these still contain nitrosamines. ${ }^{6}$ Another potential biomarker of compliance is 2-cyanoethylmercapturic acid (CEMA) ${ }^{36}$ Like CEVal, it is also a biomarker of acrylonitrile exposure and acrolein but this is often used as an efficacy endpoint in tobacco aerosol exposure studies and has a short elimination half-life of 8 hours, ${ }^{37}$ which prevents its use for long or even mid-term compliance assessment.

Given the limitations of current biomarkers used to assess compliance or lack of compliance, development of new biomarkers is essential to enable appropriate assessment of effects and cessation in an increasingly complex nicotine product use landscape. The abundance of acrylonitrile in cigarette smoke ${ }^{16}$ and the extremely low levels in emissions from THPs ${ }^{6,14}$ gives CEVal a high degree of specificity for smoking cessation in THP users. $^{23,32}$ Furthermore, it has the potential to show differences between various levels of cigarette smoke exposure ${ }^{23,38}$ and is steadily reversible upon smoking cessation. ${ }^{38}$ While it would be beneficial to have a measure that gives immediate results for participant compliance (eg, a urine or saliva dipstick test), none is currently available. Nevertheless, as shown in this analysis, percentiles could provide valuable guidance about the trajectory of non-compliance in long-term THP switching studies while results are awaited. Of note, all the percentile estimates used in this analysis are conservative, as some participants in the cessation group reported low levels of smoking in the first 30 days of the study.

We used CEVal to evaluate lack of compliance in our clinical study assessing biomarker changes in volunteers switching from smoking to a THP for up to 1 year. Based on literature we selected a threshold of $35 \mathrm{pmol} / \mathrm{g}$ globin, above which we considered indicated lack of compliance after 180 days of THP product use. ${ }^{27,32,33}$ This threshold is likely to be conservative as point estimates from quantile regression suggest $95 \%$ of concentrations at day 180 were below $25 \mathrm{pmol} / \mathrm{g}$ globin and an estimated average half-life of 30 days. Therefore, we expect to see very low levels of CEVal at the end of the THP switching study, in line with levels of $<4 \mathrm{pmol} / \mathrm{g}$ globin for most non-smokers and a mean level of $4.9 \pm 1.9 \mathrm{pmol} / \mathrm{g}$ globin reported by Pérez et al. ${ }^{38}$

Our priority in this study was identification of lack of compliance (ie, sensitivity detecting smoking volunteers) while retaining those with potentially a slower metabolism or with low level of dual use which potentially could lead to exclusive use of the THP product in the last 6 months of the study. The threshold at $35 \mathrm{pmol} / \mathrm{g}$ globin suited this purpose as more of $95 \%$ of volunteers were also identified as smokers by either of the NNAL classification criteria. These patterns appear to be in agreement with reports of biomarkers at population levels indicating high sensitivity to identify lack of compliance but lower specificity, with approximately $20 \%$ of participants classified as 'daily dual users' and 'predominant smokers' yielding low concentrations of both NNAL and CEMA. ${ }^{39}$ Threshold favouring sensitivity may have compromised the specificity of the assessment, however, it is important to highlight that there is no reason to think that excretion kinetics for NNAL and CEVAL should behave similarly among volunteers, due to metabolic differences and the variety of brands and differences in consumption at baseline. In a study where all participants 
were smoking the same control cigarettes, it was shown that consumption alone was insufficient to explain differences in CEVal between participants, additional variability would be introduced by other uncontrolled variables. ${ }^{18}$ Certainly, this relationship with consumption was not as strong as reported by Bergmark and colleagues. ${ }^{32}$ Misclassified observations based on CEVal have a mean concentration for NNAL well below those classified as not being complaint $(20.9 \mathrm{pg} / \mathrm{mL}$ vs. $101 \mathrm{pg} / \mathrm{mL})$ which could be indicative of reduction in consumption or dual use or simply suggest that perhaps NNAL could be more sensitive to sporadic cigarette consumption.

Our ROC analyses show that optimal thresholds, based of difference between sensitivity and specificity, could improve for NNAL threshold at $40 \mathrm{pg} / \mathrm{mL}$ and CEVal 35 $\mathrm{pmol} / \mathrm{g}$ globin reaching $81 \%$ sensitivity and specificity for this dataset. However, depending on specific study goals, percentiles of CEVal across time could be selected to enhance classification across time. In support of our estimates Schettgen et $\mathrm{al}^{40}$ reported a median CEVal concentration in smokers of $109 \mathrm{pmol} / \mathrm{g}$ globin and a $95^{\text {th }}$ percentile of $238 \mathrm{pmol} / \mathrm{g}$ globin (range 7-256 pmol/g globin). In 2 small studies the mean CEVal level in 14 smokers was 86.2 (range of 8.3-178) pmol/g globin in one ${ }^{38}$ and, in the other, the median in 16 smokers was 130 (range of 37-331) pmol/g globin. ${ }^{33}$ We found one study with estimated CEVal concentrations higher than ours (mean $252 \pm 22 \mathrm{pmol} / \mathrm{g}$ globin in 18 smokers smoking one pack of cigarettes per day), ${ }^{41}$ but the type of cigarettes smoked was not reported, which could explain the difference in the estimates.

Our approach is subject to some limitations. Firstly, although this is probably the largest study to date assessing CEVal concentrations over time, the sample size is still small to appropriately characterise different populations and products. Secondly, controlled studies would be required to assess the relationship between consumption and product type and CEVal concentrations, as well as gaining further understanding of the effect of sporadic smoking in relation to the time of sample collection. Predictability of CEVal might be improved by using more complex modelling or machine learning techniques incorporating demographic factors, consumption and even perhaps a combination of several biomarkers to enhance classification accuracy.

\section{IMPLICATIONS FOR TOBACCO REGULATION}

Self-reported compliance in smoking cessation and switching studies can be highly unreliable. Biomarkers of compliance may prove to be useful tools to verify nicotine product use status in clinical and observational studies. However, currently available biomarkers of compliance may not be suitable to assess changes over longer periods of time than a few days or to clearly differentiate smoking from some nicotine product categories like THPs.

A new generation of biomarkers of compliance is required, specific to the new generation of nicotine products. Methodological validation and standardisation could allow robust assessment of effects across clinical and observational studies while promoting comparability between studies. Highly accurate biomarkers of compliance would aid both product manufactures and regulatory agencies to dissociate product effects from misreported product use.

We have presented CEVal as a potential candidate biomarker of compliance which, due to its relatively long half-life and specificity to tobacco smoke, could be useful to assess sustained smoking cessation or switching to alternative products. A greater effort from all parties involved is required to validate the use of this and other biomarkers and establishing protocols for their use. 


\section{Human Subjects Statement}

The study protocol was approved by an independent ethics national research committee (NHS Health Research Authority, Wales Research Ethics Committee 2 (reference number: 17/WA/0212)) and participants provided written informed consent before data collection began.

\section{Conflict of Interest Statement}

At the time of the study, OMC, MM, NG, GH, and JM were employees of British American Tobacco. This work was fully funded by British American Tobacco (Investments) Limited.

\section{References}

1. US Department of Health and Human Services. The Health Consequences of Smoking: 50 Years of Progress: a Report of the Surgeon General. Atlanta: Department of Health and Human Services, Centers for Disease Control and Prevention, National Center for Chronic Disease Prevention and Health Promotion, Office on Smoking and Health; 2014.

2. Institute of Medicine. Clearing the Smoke - Assessing the Science Base for Tobacco Harm Reduction. Washington. D.C.: The National Academies Press; 2001.

3. Stratton K, Shetty P, Wallace R, et al. Clearing the smoke: the science base for tobacco harm reduction--executive summary. Tob Control. 2001;10:189-95

4. Royal College of Physicians. Nicotine without Smoke. Tobacco Harm Reduction. A Report by the Tobacco Advisory Group of the Royal College of Physicians. London: Royal College of Physicians; 2016.

5. Eaton D, Jakaj B, Forster M, et al. Assessment of tobacco heating product THP1.0. Part 2: Product design, operation and thermophysical characterisation. Regul Toxicol Pharmacol. 2018;93:4-13.

6. Forster M, Fiebelkorn S, Yurteri C, et al. Assessment of novel tobacco heating product THP1.0. Part 3: Comprehensive chemical characterisation of harmful and potentially harmful aerosol emissions. Regul Toxicol Pharmacol. 2018;93:14-33.

7. Bentley MC, Almstetter M, Arndt D, et al. Comprehensive chemical characterization of the aerosol generated by a heated tobacco product by untargeted screening. Anal Bioanal Chem. 2020;412(11):2675-2685.

8. Uchiyama S, Noguchi M, Takagi N, et al. Simple determination of gaseous and particulate compounds generated from heated tobacco products. Chem Res Toxicol. 2018;31(7):585-593.

9. Gale N, McEwan M, Eldridge AC, et al. Changes in biomarkers of exposure on switching from a conventional cigarette to tobacco heating products: a randomized, controlled study in healthy Japanese subjects. Nicotine Tob Res. 2019;21(9):1220-1227.

10. Newland N, Lowe FJ, Camacho OM, et al. Evaluating the effects of switching from cigarette smoking to using a heated tobacco product on health effect indicators in healthy subjects: study protocol for a randomized controlled trial. Intern Emerg Med. 2019;14(6):885-898.

11. Haziza C, de La Bourdonnaye G, Donelli A, et al. Reduction in exposure to selected harmful and potentially harmful constituents approaching those observed upon smoking abstinence in smokers switching to the menthol tobacco heating system 2.2 for 3 months (part 1). Nicotine Tob Res. 2020;22(4):539-548.

12. Benowitz NL, Bernert JT, Foulds J, et al. Biochemical verification of tobacco use and abstinence: 2019 update. Nicotine Tob Res. 2020;22(7):1086-1097. 
13. Benowitz NL, Nardone N, Jain S, et al. Comparison of urine 4-(methylnitrosamino)-1(3)pyridyl-1-butanol and cotinine for assessment of active and passive smoke exposure in urban adolescents. Cancer Epidemiol Biomarkers Prev. 2018;27(3):254-261.

14. Schaller JP, Keller D, Poget L, et al. Evaluation of the Tobacco Heating System 2.2. Part 2: Chemical composition, genotoxicity, cytotoxicity, and physical properties of the aerosol. Regul Toxicol Pharmacol. 2016;81(suppl 2):S27-S47.

15. Goniewicz ML, Havel CM, Peng MW, et al. Elimination kinetics of the tobacco-specific biomarker and lung carcinogen 4-(methylnitrosamino)-1-(3-pyridyl)-1-butanol. Cancer Epidemiol Biomarkers Prev. 2009;18(12):3421-3425.

16. Eldridge A, Betson T, Gama MV, et al. Investigation of number of replicate measurements required to meet cigarette smoke chemistry regulatory requirements measured under Canadian intense smoking conditions. Regul Toxicol Pharmacol. 2019;107:104402.

17. St Helen G, Jacob P, 3rd, Peng M, et al. Intake of toxic and carcinogenic volatile organic compounds from secondhand smoke in motor vehicles. Cancer Epidemiol Biomarkers. Prev 2014;23(12):2774-2782.

18. Scherer G, Newland K, Papadopoulou E, et al. A correlation study applied to biomarkers of internal and effective dose for acrylonitrile and 4-aminobiphenyl in smokers. Biomarkers. 2014;19(4):291-301.

19. Gregg EO, Minet E, McEwan M. Urinary biomarkers of smokers' exposure to tobacco smoke constituents in tobacco products assessment: a fit for purpose approach. Biomarkers. 2013;18(6):467-486.

20. Luo X, Carmella SG, Chen M, et al. Urinary cyanoethyl mercapturic acid, a biomarker of the smoke toxicant acrylonitrile, clearly distinguishes smokers from non-smokers. Nicotine Tob Res. 2020;22(10):1744-1747.

21. Vesper HW, Caudill SP, Osterloh JD, et al. Exposure of the U.S. population to acrylamide in the National Health and Nutrition Examination Survey 2003-2004. Environ Health Perspect. 2010;118(2):278-283.

22. Van Nieuwenhuyse A, Fierens S, De Smedt T, et al. Acrylonitrile exposure assessment in the emergency responders of a major train accident in Belgium: a human biomonitoring study. Toxicol Lett. 2014;231(3):352-359.

23. Kütting B, Uter W, Drexler H. The association between self-reported acrylamide intake and hemoglobin adducts as biomarkers of exposure. Cancer Causes Control. 2008;19(3):273-281.

24. Schettgen T, Müller J, Ferstl C, et al. Haemoglobin adducts of ethylene oxide (N-(2hydroxyethyl)valine), propylene oxide (N-(2-hydroxypropyl)valine), acrylonitrile (N-(2cyanoethyl)valine), acrylamide (N-(2-carbonamide ethyl)valine) and glycidamide (N-(2hydroxy-2-carbonamide ethyl)valine). Biomonitoring Methods. 2015;2016;1(1):473-506.

25. Van Sittert NJ. N-2-Cyanoethylvaline, N-2-Hydroxyethylvaline, N-Methylvaline (as evidence of exposure to acrylonitrile, ethylene oxide as well as methylating agents). In: Greim H, editor. Biomonitoring Methods. Weinheim: Wiley-VCH 1996: 181-219.

26. Kavvadias D, Scherer G, Cheung F, et al. Determination of tobacco-specific Nnitrosamines in urine of smokers and non-smokers. Biomarkers. 2009;14(8):547-553.

27. Camacho O, Hedge A, Lowe F, et al. Statistical analysis plan for "A randomised, controlled study to evaluate the effects of switching from cigarette smoking to using a tobacco heating product on health effect indicators in healthy subjects". Contemp Clin Trials Commun. 2020;17:100535.

28. Koenker R, d'Orey V. Computing regression quantiles. Applied Statistics 1993;43:410414 
29. Carmella SG, Han S, Fristad A, et al. Analysis of total 4-(methylnitrosamino)-1-(3pyridyl)-1-butanol (NNAL) in human urine. Cancer Epidemiol Biomarkers Prev. 2003;12(11 Pt 1):1257-1261.

30. Office on Smoking and Health. The Health Consequences of Involuntary Exposure to Tobacco Smoke: A Report of the Surgeon General. Atlanta: Centers for Disease Control and Prevention 2006.

31. Centers for Disease Control and Prevention. 201712 June. Biomonitoring Summary. 4(Methylnitrosamino)-1-(3-pyridyl)-1-butanol (NNAL). https://www.cdc.gov/biomonitoring/NNAL_BiomonitoringSummary.html. Accessed March 23, 2021.

32. Bergmark E. Hemoglobin adducts of acrylamide and acrylonitrile in laboratory workers, smokers and nonsmokers. Chem Res Toxicol. 1997;10(1):78-84.

33. Schettgen T, Rossbach B, Kütting B, et al. Determination of haemoglobin adducts of acrylamide and glycidamide in smoking and non-smoking persons of the general population. Int J Hyg Environ Health. 2004;207(6):531-539

34. Haziza C, de La Bourdonnaye G, Donelli A, et al. Reduction in exposure to selected harmful and potentially harmful constituents approaching those observed upon smoking abstinence in smokers switching to the menthol tobacco heating system 2.2 for three months (Part 1). Nicotine Tob Res. 2020;22(4):539-548.

35. Hecht SS, Yuan JM, Hatsukami D. Applying tobacco carcinogen and toxicant biomarkers in product regulation and cancer prevention. Chem Res Toxicol. 2010;23(6):1001-1008.

36. Lüdicke F, Ansari SM, Lama N, et al. Effects of switching to a heat-not-burn tobacco product on biologically relevant biomarkers to assess a candidate modified risk tobacco product: a randomized trial. Cancer Epidemiol Biomarkers Prev. 2019;28(11):19341943.

37. Jakubowski M, Linhart I, Pielas G, et al. 2-Cyanoethylmercapturic acid (CEMA) in the urine as a possible indicator of exposure to acrylonitrile. Br J Ind Med. 1987;44(12):834840.

38. Pérez HL, Segerbäck D, Osterman-Golkar S. Adducts of acrylonitrile with hemoglobin in nonsmokers and in participants in a smoking cessation program. Chem Res Toxicol. 1999; 12(10):869-873.

39. Smith DM, Christensen C, van Bemmel D, et al. Exposure to nicotine and toxicants among dual users of tobacco cigarettes and e-cigarettes: Population Assessment of Tobacco and Health (PATH) study, 2013-2014. Nicotine Tob Res. 2021;23(5):790-797.

40. Schettgen T, Weiss T, Drexler H, et al. A first approach to estimate the internal exposure to acrylamide in smoking and non-smoking adults from Germany. Int J Hyg Environ Health. 2003;206(1):9-14.

41. Fennell TR, MacNeela JP, Morris RW, et al. Hemoglobin adducts from acrylonitrile and ethylene oxide in cigarette smokers: effects of glutathione $S$-transferase T1-null and M1null genotypes. Cancer Epidemiol Biomarkers Prev. 2000;9(7):705-712. 
